\title{
TRATAMIENTO Y DAÑO TÉRMICO DE ARTEFACTOS LÍTICOS EN LOS COMPONENTES TEMPRANOS DEL SITIO CASA DEL MINERO 1, SANTA CRUZ, ARGENTINA
}

\author{
HEAT TREATMENT AND HEAT DAMAGE OF LITHIC ARTIFACTS \\ FROM THE EARLY COMPONENTS OF SITE CASA DEL MINERO 1, \\ SANTA CRUZ, ARGENTINA
}

\begin{abstract}
Ariel D. Frank ${ }^{1}$
En este trabajo analizo el conjunto lítico de dos componentes del sitio Casa del Minero 1 -ubicado en la localidad arqueológica La María, Meseta Central de Santa Cruz a 155 msm - con cronologías de entre 11.000 y 10.250 años antes del presente. Evalúo las causas de termoalteración de estos materiales distinguiendo procesos intencionales de accidentales.

Para ello, estudio sus características tipológicas y los indicadores macroscópicos de termoalteración presentes: su ubicación en las distintas caras de las piezas y el análisis de las secuencias de producción permite identificar el tratamiento térmico y diferenciarlo de otras termoalteraciones. Asimismo, analizo la distribución de estas piezas y su vinculación con los fogones.

Los restos tratados, especialmente en sílex rojo, abundan en la Unidad 4; hay numerosas lascas de adelgazamiento bifacial con lustre térmico. En esta unidad el tratamiento térmico se habría realizado sobre formas base. En la Unidad 3C hay escasos artefactos termoalterados. La alta variedad de materias primas posiblemente tratadas y su dispersión indicarían que el tratamiento térmico y talla de estos artefactos se realizó fuera del área excavada.

La termoalteración de las piezas dañadas no se dio durante el tratamiento térmico. Son escasas y proceden de cuadrículas con fogones, por lo que probablemente este daño se haya producido por su caída accidental en estos.

Palabras claves: tratamiento térmico, daño térmico, Santa Cruz, cazadores recolectores, manejo del fuego.
\end{abstract}

In this paper, I analyze the lithic assemblage of two components from Casa del Minero 1 site -La María archaeological locality, in the Central Plateau of Santa Cruz. This site is 155 masl, with dates ranging from 11,000 to 10,250 years before present. I study the potential causes of the thermal alteration seen in these materials, distinguishing intentional from accidental causes.

To that end, I study typological characteristics and the macroscopic indicators of thermal alteration in the assemblage: their location on the flakes and analysis of the production sequences allow me to identify heat treatment as well as other thermal alteration processes. In addition, I examined the distribution of these artifacts to determine whether they were located near hearths.

Heat treated remains, especially those on red flint, are plentiful in Unit 4; many bifacial thinning flakes show thermal luster. I argue that heat treatment was conducted on blanks. In Unit $3 C$ there are few artifacts altered by heat. The wide variety and high dispersion of raw materials that possibly received this form of treatment may indicate that this was performed in an area that has not been excavated.

The thermal alteration of damaged artifacts was not due to heat treatment. These are very scarce and were found in grids that had hearths, and as such I suggest that the thermal damage was caused by an accidental fall into the hearths.

Key words: Heat treatment, thermal damage, Santa Cruz, hunter-gatherers, fire management.

Se entiende por tratamiento térmico a toda exposición de materiales al calor producido por el fuego con el objetivo de modificar sus propiedades intrínsecas para propósitos particulares. Dentro de esta categoría amplia se incluye el tratamiento térmico de artefactos líticos, que consiste en exponer material lítico a la acción del calor controlado de fogones, a fin de optimizar sus cualidades para la talla, pues la temperatura cambia las propiedades de las rocas en su estructura cristalográfica. Este proceso genera cambios en color y lustre, además de fracturas u otras alteraciones específicas y diagnósticas en las superficies artefactuales (Inizan y Tixier 2000; Luedtke 1992).

Esta técnica es parte de la producción de instrumentos de uso cotidiano. Altera algunas de las variables que se conjugan en el proceso de talla. Por ejemplo, disminuye la fuerza necesaria para extraer lascas, permitiendo generar productos más largos y con morfologías controladas; además, reduce las

1 Departamento Científico de Arqueología, FCNyM, UNLP/ CONICET. Paseo del Bosque s/n (1900). La Plata. Buenos Aires, Argentina. frank.ariel@gmail.com 
probabilidades de ocasionar fracturas en charnela. El control de la temperatura que necesita esta técnica destaca como la cualidad más importante durante su aplicación en pos de los resultados buscados.

El tratamiento térmico fue una técnica utilizada por los grupos que habitaron la Patagonia a lo largo del tiempo (Hermo 2008; Nami 1992; Paunero 2009; Stadler 2002; entre otros). Sin embargo, aun no se sabe con seguridad el modo en que ésta fue llevada a cabo, ni en qué etapa del proceso de producción de artefactos líticos. Existen evidencias en diferentes partes del mundo de tratamiento térmico de núcleos (Collins y Fenwick 1974; Gassin et al. 2006; Inizan et al. 1999; Inizan y Tixier 2000; Rick y Chappell 1983; Terradas y Gibaja 2001) y de formas base (Collins y Fenwick 1974; Mandeville 1973; Patterson 1984; Rick y Chappell 1983; Schindler et al. 1982). También en la Patagonia se ha propuesto el tratamiento térmico para ambos tipos de artefactos (Paunero 2009; Stadler 2002), pero las evidencias son aún muy escasas como para poder afirmarlo fehacientemente.

Otras situaciones de alteración térmica involuntaria o no dirigida podrían encuadrarse como circunstancias accidentales: la caída casual de una pieza en un fogón; el descarte de artefactos en estas estructuras de combustión como forma de mantenimiento del espacio o en el marco de rituales mortuorios; o la acción de incendios naturales o de fogones encendidos después de la depositación de estos elementos (Clemente Conte 1995; Larsson 2000; Musters 2003; Stadler 2002).

Así, resulta interesante poder diferenciar en qué casos una pieza resultó termoalterada por un tratamiento térmico con fines tecnológicos, y en cuáles dicha alteración se debió a otras causas.

En este trabajo analizo el conjunto lítico de los componentes tempranos del sitio Casa del Minero 1 (CDM1), focalizando en los artefactos termoalterados. Estudio sus características tipológicas, el tipo de indicador de termoalteración presente y la distribución de estas piezas dentro del sitio. El objetivo es evaluar cuáles fueron las causas de la alteración térmica de estos materiales, buscando diferenciar causas intencionales de accidentales.

\section{Caracterización del Sitio}

CDM1 se ubica en el sector Cañadón de la Mina, Localidad Arqueológica La María, Meseta Central de Santa Cruz, Argentina, a 155 msm (Figura 1).
Se localiza a $118 \mathrm{~km}$ al sur de Los Toldos, a $45 \mathrm{~km}$ al sur de la localidad Cerro Tres Tetas y a $9 \mathrm{~km}$ al suroeste de El Ceibo.

Entre los años 2000 y 2004 se excavaron $15,32 \mathrm{~m}^{2}$ del sitio $-22,5 \%$ de su superficie-mediante una técnica de decapage con intención de identificar unidades de depositación discernibles estratigráficamente (Paunero et al. 2007). Esta cueva es un sitio multicomponente con evidencias de ocupaciones humanas que van desde el Pleistoceno final hasta inicios del siglo XX (Paunero et al. 2007). Las unidades estratigráficas identificadas con restos culturales correspondientes al poblamiento temprano son las unidades 4 y $3 \mathrm{C}$ (Tabla 1) (Paunero et al. 2007). Estos dos componentes están separados por una clara línea de rocas de derrumbe que sella la ocupación más temprana. El fechado de ambas unidades nos da una edad máxima y una edad mínima para este episodio de derrumbe.

Estas unidades corresponden a ocupaciones tempranas en el poblamiento de la región, con características diagnósticas de fase colonizadora e incluyen el registro de tres especies de camélidos: el guanaco y dos extintos (Hemiauchenia paradoxa y Lama (vicugna) gracilis), dos variedades de cánidos y Rhea sp. El análisis de los conjuntos óseos muestra actividades como procesamiento primario y consumo, según las evidencias de fracturas helicoidales y marcas de corte, pero otros indicadores remiten al trabajo en cuero y en hueso, a la elaboración de diferentes bienes y a la producción de intermediarios para el procesamiento secundario de los productos derivados (Paunero et al. 2007).

Con relación al conjunto lítico, se observa un buen conocimiento de las materias primas locales y el uso de estrategias tecnológicas vinculadas a la oferta de recursos líticos del ambiente. Presenta poca diversidad de materias primas, predominando el sílex rojo de procedencia local y buena calidad para la talla (Skarbun et al. 2007). La mayoría de los instrumentos son expeditivos y se habrían terminado de formatizar en el sitio, sin registrarse evidencias de las primeras etapas de producción, que se realizaron en otro sector, como las canteras o sitios al aire libre cercanos a la cueva (Frank et al. 2007). También se registran evidencias de bifacialidad y laminaridad (Skarbun et al. 2007).

Ciertas características del conjunto lítico remiten a la aplicación de la técnica de tratamiento térmico. De esta manera, me interesa evaluar si realmente se llevó a cabo esta práctica, y si es así en qué tipo 


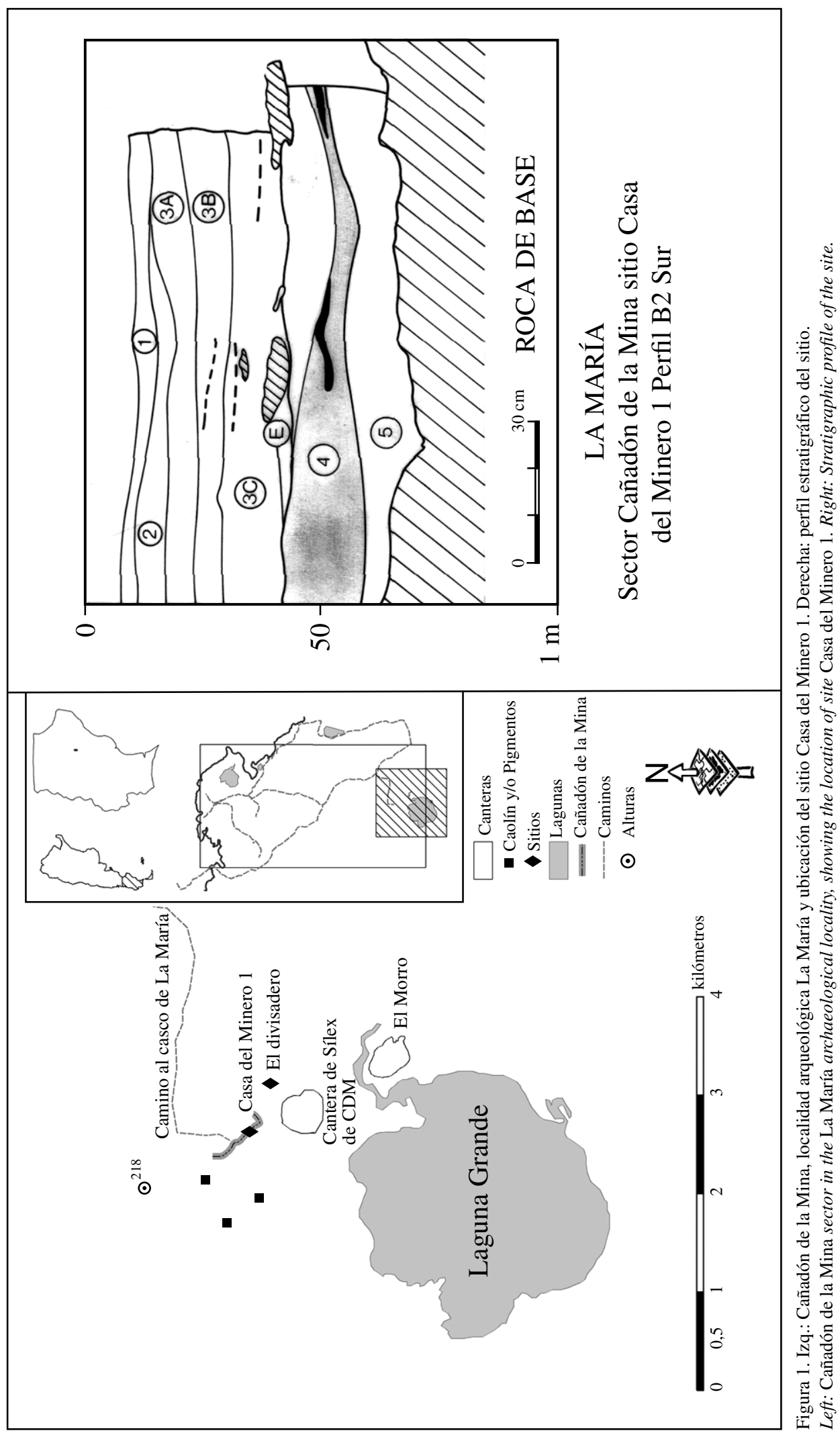


Tabla 1. Fechados radiocarbónicos correspondientes a las ocupaciones de Pleistoceno final en CDM1. Unidades 4 y 3C. Todos los fechados calibrados a 2 sigmas con el programa Calib Rev 6.0.1. de Reimer et al. 2009. AA: Arizona.

Radiocarbon dates from the final Pleistocene occupations at CDM1. Units 4 and 3C. All dates were calibrated in 2 sigmas using Calib Rev 6.0.1., Reimer et al. 2009. AA: Arizona.

\begin{tabular}{|c|c|c|c|c|}
\hline Unidad & Edad radiocarbónica años a.p. & $\begin{array}{l}\text { Número de } \\
\text { laboratorio }\end{array}$ & $\begin{array}{l}\text { Material } \\
\text { fechado }\end{array}$ & cal. 2 sigma \\
\hline $3 \mathrm{C}$ & $10.250 \pm 110$ & AA 45705 & Carbón & $\begin{array}{c}10.563-10.554 \text { a.C. }(p=0,002012) 10.472-9.649 \\
\text { a.C. }(p=0,964652) 9.606-9.526 \text { a.C }(p=0,024361) \\
9.491-9.459 \text { a.C. }(p=0,008975)\end{array}$ \\
\hline \multirow{2}{*}{4} & $10.967 \pm 55$ & AA 37208 & Carbón & $\begin{array}{l}11.118-11.057 \text { a.C. }(p=0,061438) \\
11.037-10.711 \text { a.C. }(p=0,938562)\end{array}$ \\
\hline & $10.999 \pm 55$ & AA 37207 & Carbón & 11.131 - 10.742. a.C. \\
\hline
\end{tabular}

de artefactos. Por otro lado, es necesario analizar cuál fue la causa de la alteración térmica de estos artefactos en caso que se compruebe que no se había aplicado este procedimiento.

\section{Metodología}

Inicialmente, he realizado una clasificación del material lítico procedente de estas unidades, que consistió en una clasificación tipológica basada en los criterios construidos por nuestro equipo (Skarbun 2009), complementados con elementos de la bibliografía específica (Aschero 1975; Cardich y Flegenheimer 1978; Cardich et al. 1981-1982; Orquera y Piana 1986). He incluido en dicha clasificación los rasgos de termoalteración presentes en las piezas, diferenciando su ubicación en la cara dorsal o ventral. Las observaciones se realizaron a ojo desnudo y con lupa (10x).

La identificación de los rasgos de alteración térmica se basó en la bibliografía existente para esta temática (Bordes 1969; Cattáneo et al. 19971998; Clemente Conte 1995; Collins y Fenwick 1974; Mandeville 1973; Nami et al. 2000; Purdy y Brooks 1971; Terradas y Gibaja 2001; entre otros), así como en los patrones experimentales de referencia construidos en fases previas de mi investigación (Cueto y Frank 2008; Frank 2009; Frank et al. 2009). Tales rasgos son:

Hoyuelos: También llamados levantamientos térmicos. Son negativos de forma cónica que se producen en el cuerpo de la lasca. El desprendimiento que provoca este hoyuelo es denominado cono de desprendimiento.

Escamaciones: Son fracturas en forma de medialuna, producto del desprendimiento incompleto de los conos. Puede hallárselas aisladas o en grupo.
Rugosidad: Se caracteriza por ser una superficie sumamente irregular y áspera producto del desprendimiento de muy pequeños fragmentos de la pieza.

Agrietamientos: Son fisuras irregulares, de morfología variable, superficiales o profundas. En algunos casos puede formar un reticulado conocido como craquelado.

Cambio de color: El material lítico puede cambiar su coloración al ser expuesto a altas temperaturas si contiene minerales que, como el hierro, reaccionen con una atmósfera oxidante al ser calentados (Purdy y Brooks 1971; Schindler et al. 1982). El cambio de color no ocurrirá en ausencia de minerales adecuados o si las rocas son calentadas en un ambiente reductor.

Lustre: Es un brillo graso que se observa en el interior de la pieza una vez que ésta fue retocada con posterioridad al tratamiento térmico, o como consecuencia de fracturas o desprendimientos.

Pátina: Es una cubierta blanquecina superficial, poco frecuente, al menos en las experimentaciones realizadas por el autor. Se asocian por lo general a temperaturas elevadas (Clemente Conte 1995).

Los primeros cuatro indicadores (hoyuelos, escamaciones, rugosidad y agrietamientos) son indicadores de daño térmico, es decir, son fracturas generadas por la exposición de las piezas al calor. Ocurren en la superficie externa de la pieza, aunque en algunos casos esta queda totalmente destruida y no se puede reconocer la superficie original. $\mathrm{Si}$ bien en algunas oportunidades estas fracturas no impiden que se prosiga con la talla, en otras la pieza se vuelve inutilizable. Aunque en baja proporción, pueden observarse en algunos casos de tratamiento térmico, puesto que de ser predominantes, el tratamiento térmico habría resultado fallido. 
Por su parte, el cambio de color, el lustre y la pátina también son alteraciones térmicas pero no conjugan un cambio en las propiedades para la talla por sí mismas. Así, se generan diferentes expectativas de acuerdo al hallazgo de los diversos indicadores en diferentes tipos de piezas.

Una vez realizada la clasificación, se analizó qué tipo de resto presenta evidencias de termoalteración, es decir, si son núcleos, instrumentos o bien productos de talla ${ }^{1}$. En el caso de los productos de talla, interesa saber a qué estadio de reducción corresponden (descortezamiento -1 , talla -2 o formatización final -3; sensu Skarbun et al. 2007). También he evaluado la ubicación de estos rasgos en relación a la morfología general de las piezas, es decir, si se presentan en la cara dorsal, en la ventral o en ambas.

La combinación del estudio del tipo de resto con termoalteración y la ubicación de las termoalteraciones en las piezas es relevante para asignar la causa de su alteración, y en el caso del tratamiento térmico permite evaluar en qué momento del proceso de confección de los instrumentos este procedimiento pirotecnológico fue llevado a cabo. Se podrían resumir las expectativas de hallazgo de rasgos de alteración térmica en piezas tratadas de la siguiente manera:

Lustre: Se encontrará lustre en los negativos de lascado de la pieza tratada originalmente, y en la cara dorsal y ventral de las piezas extraídas de ella (excepto las primeras lascas extraídas que solo tendrán lustre en su cara ventral).

Color: El cambio de color se detectará en la pieza originalmente expuesta al calor, y en ambas caras de las primeras piezas extraídas de ella.

Pátina: La pátina, de estar presente, se presentará en la pieza originalmente expuesta al calor, y en una o ambas caras de las piezas inicialmente extraídas de ella.

Daño térmico: Sólo se hallará daño térmico en la cara dorsal de la pieza tratada o en piezas extraídas de ella que contengan parte de su superficie original. No se encontrará daño en la cara ventral de piezas tratadas térmicamente excepto si es la pieza originalmente expuesta al fuego (núcleo o forma base).

Vale aclarar que este es un modelo simplificado y que es necesario un análisis particular de cada caso para poder reconocer procesos de tratamiento térmico en los conjuntos líticos.
Por otra parte, en los casos de daño térmico por causas no relacionadas al tratamiento pirotecnológico, las expectativas difieren. En estas ocasiones, se puede observar daño térmico y cambio de coloración en cualquiera de las dos caras, siendo predominante en la cara que estuvo más cercana a la fuente de calor. El lustre no se observará excepto en los hoyuelos y en la cara ventral de los conos de desprendimiento. La pátina puede estar presente, pero siempre en baja proporción.

Finalmente, he realizado una aproximación inicial a la distribución de los artefactos líticos termoalterados, prestando atención a su relación con los fogones, para determinar si existieron eventos de alteraciones no intencionales y postdepositacionales de los materiales. En el caso de las piezas dañadas, el análisis de su distribución en conjunto con sus características tipológicas y tecnológicas puede dar información útil para analizar las causas de su termoalteración. Basándome en mis experimentaciones (Cueto y Frank 2008; Frank 2009; Frank et al. 2009), así como en las propuestas de otros autores, postulo las siguientes expectativas:

- Para los casos producidos por incendios naturales o fogones encendidos durante ocupaciones posteriores, espero que las piezas termoalteradas se concentren inmediatamente por debajo de la fuente de combustión, a una distancia máxima de $10 \mathrm{~cm}$. Se espera que no exista selección de tamaños ni de formas entre los elementos alterados (Stadler 2002).

- Para los casos de descarte de piezas dentro del fogón, espero una concentración de piezas dentro del fogón y en menor medida en sus alrededores. Existen al menos dos posibles causas para el descarte de las piezas en un fogón. En casos de limpieza y mantenimiento del espacio, espero que no exista una selección de grupos tipológicos entre los elementos descartados en la estructura de combustión. Si bien casos como el mencionado son difíciles de encontrar en la literatura arqueológica y no son especialmente esperables en tanto que si esta conducta es repetitiva termina colmatando el fogón, considero conveniente tenerlo en cuenta puesto que es una alternativa por la cual las piezas pueden resultar termoalteradas. En casos vinculados a la destrucción de piezas en el fuego durante rituales mortuorios como los mencionados por Musters (2003) espero que 
los elementos termoalterados sean instrumentos terminados.

- Para los casos de caída accidental de piezas en un fogón espero que estas se concentren tanto dentro como en los alrededores del fogón, pero que, a diferencia de los casos anteriores, la proporción de elementos termoalterados sea baja. Espero que los elementos termoalterados sean principalmente pequeños (Nakazawa 2007).

En cuanto a los materiales tratados térmicamente, el análisis distribucional busca evaluar si su talla posterior se dio en los alrededores de los fogones o bien en otras áreas.

Esta sucesión de pasos permite evaluar el grado de alteración térmica del conjunto lítico de CDM1 y reconocer qué actividades del pasado, relativas al fuego, intencionales o no, han actuado en su configuración actual. Además, ayuda a identificar si se aplicó la técnica del tratamiento térmico en artefactos líticos y evaluar en qué casos se llevó a cabo.

\section{Resultados}

He realizado la observación, clasificación y análisis de los conjuntos líticos del sitio arqueológico Casa del Minero 1 (CDM1), con énfasis en los componentes finipleistocénicos.

\section{Unidad 4}

La Unidad 4 presenta abundantes productos de talla (XT, $n=1.229)$, escasos instrumentos (I, $n=11)$ y ningún núcleo $(\mathrm{N})$. Abundan los productos de talla de formatización final $(n=540)$ y son escasos los de descortezamiento $(n=39)$ (Tabla 2). La materia prima predominante es el sílex $(69,84 \%, \mathrm{n}=866)$, en especial el de color rojo de procedencia local $(48,23 \%$ del total del conjunto, $n=598)$ (Tabla 3$)$.
Tabla 2. Estructura general del conjunto. Unidades 4 y $3 \mathrm{C}$. General structure of the assemblage. Units 4 and $3 C$.

\begin{tabular}{ccrr}
\hline Grupo & Estadio & Unidad 4 & Unidad 3C \\
\hline I & - & 11 & 7 \\
XT & 1 & 39 & 27 \\
& 2 & 271 & 381 \\
& 3 & 540 & 233 \\
& Indet. & 379 & 549 \\
Total & & 1.240 & 1.197 \\
\hline
\end{tabular}

Hay 453 artefactos líticos con probables alteraciones térmicas. Esto representa, sobre un total de 1.240 artefactos líticos recuperados, el 36,5\% del conjunto. De estos 453 artefactos termoalterados, 44 presentan evidencias de daño térmico, mientras que los 409 elementos restantes muestran únicamente cambios en el color y/o lustre.

En cuanto a los restos dañados (44), sus características me llevan a descartar, en la mayoría de ellos, el daño producto del tratamiento térmico. Por un lado, los elementos son muy pequeños, lo que los vuelve poco factibles de ser utilizados como formas base: en todos los casos las piezas son menores a 3,5 cm de longitud; además en su mayoría $(70,5 \%)$ corresponden a esquirlas, microesquirlas $\mathrm{y}$ fragmentos de talla $(\mathrm{n}=31$, Tabla 4). También hay que remarcar que, de las 44 piezas con daño térmico, $24(54,5 \%)$ presentan daños en ambas caras, por lo que esta alteración se produjo después de haber sido extraídas del soporte original. Lo mismo puede decirse de los 14 restos dañados exclusivamente en su cara ventral (Tabla 5). Únicamente aquellas esquirlas dañadas sólo en su cara dorsal (seis) pueden ser producto del tratamiento térmico, es decir, que provengan de una pieza que fue tratada térmicamente y posteriormente tallada, arrastrando estas esquirlas la parte que resultó dañada durante el procedimiento.

Tabla 3. Artefactos por materia prima. Unidades 4 y 3C. Calc. $=$ Calcedonia. Ignim. $=$ Ignimbrita. Obs. $=$ Obsidiana. Marr. $=$ Marrón. Amar. $=$ Amarillo. TBS $=$ Toba silicificada. Xil. $=$ Xilópalo.

Artifacts according to raw material. Units 4 and 3C. Calc. $=$ Chalcedony. Ignim. $=$ Ignimbrite. Obs. $=$ Obsidian . Marr. $=$ Brown. Amar. $=$ Yellow. $T B S=$ Silicified tuff. Xil $=$ Silicified Wood .

\begin{tabular}{|c|c|c|c|c|c|c|c|c|c|c|c|c|}
\hline \multirow[b]{2}{*}{ Unidad } & \multirow[b]{2}{*}{ Calc. } & \multirow[b]{2}{*}{ Ignim. } & \multirow[b]{2}{*}{ Indet. } & \multirow[b]{2}{*}{ Obs. } & \multicolumn{4}{|c|}{ Sílex } & \multirow[b]{2}{*}{ Toba } & \multirow[b]{2}{*}{ TBS } & \multirow[b]{2}{*}{ Xil. } & \multirow[b]{2}{*}{ Total } \\
\hline & & & & & Rojo & Marr. & Amar. & Otros & & & & \\
\hline 4 & 251 & 1 & 72 & 1 & 598 & 170 & 49 & 49 & 3 & 2 & 44 & 1.240 \\
\hline $3 C$ & 69 & - & 15 & - & 928 & 93 & 50 & 32 & - & 6 & 4 & 1.197 \\
\hline
\end{tabular}


Tabla 4. Cantidad de artefactos con daño térmico por clase. Unidades 4 y 3 C. LAS = Laminilla. ESQ = Esquirla.

FT = Fragmento de Talla. $\mathrm{L}=$ Lasca. MESQ = Microesquirla. Quantity of artifacts with heat damage according to its class. Units 4 and $3 C$. LAS = Bladelet. ESQ $=$ Chip. $F T=$ Fragment. $L=$ Flakes. $M E S Q=$ Microchip.

\begin{tabular}{ccc}
\hline Clase & Unidad 4 & Unidad 3C \\
\hline LAS & 1 & - \\
ESQ & 8 & 15 \\
FT & 8 & 7 \\
L & 12 & 7 \\
MESQ & 15 & 5 \\
Total & 44 & 34 \\
\hline
\end{tabular}

Tabla 5. Piezas con rasgos de alteración térmica. Unidad 4. No $=$ Sin daño térmico pero con alteraciones del tipo lustre, color y pátina.

Artifacts with indications of thermal alteration.

Unit 4. No = Without heat damage but with other alterations such as luster, color and patina.

\begin{tabular}{cccc}
\hline \multirow{2}{*}{ Daño ventral } & \multicolumn{2}{c}{ Daño dorsal } & Total \\
\cline { 2 - 3 } & No & Sí & \\
\hline No & 409 & 6 & 415 \\
Sí & 14 & 24 & 38 \\
Total & 423 & 30 & 453 \\
\hline
\end{tabular}

$\mathrm{Al}$ analizar su procedencia, se observa que las piezas con daño provienen mayormente de cuadrículas en las que se han registrado fogones (Tabla 6 y Figura 2). En algunos casos estos restos fueron recuperados dentro de las estructuras de combustión. Las características de este subconjunto apuntan a que la termoalteración se haya producido por la caída accidental de lascas en los fogones al momento de confección de instrumental lítico, o posteriormente. Rechazo un descarte intencional de las piezas líticas a los fogones por la baja cantidad de elementos dañados con relación al total. Además en casos de mantenimiento del espacio sería esperable encontrar mayor cantidad de restos dañados, que abarquen un mayor espectro de tamaños y de grupos tipológicos; en casos relacionados a rituales mortuorios espero encontrar daño en instrumentos terminados, hecho que no se ha dado. En este sentido, las características del sitio son de funciones domésticas, por lo que esta posibilidad resulta de por sí poco probable. También descarto la posibilidad de una alteración por fogones encendidos en ocupaciones posteriores, puesto que las cuadrículas que cuentan con mayor cantidad de piezas con daños no presentan fogones en la Unidad 3C (Figura 2).

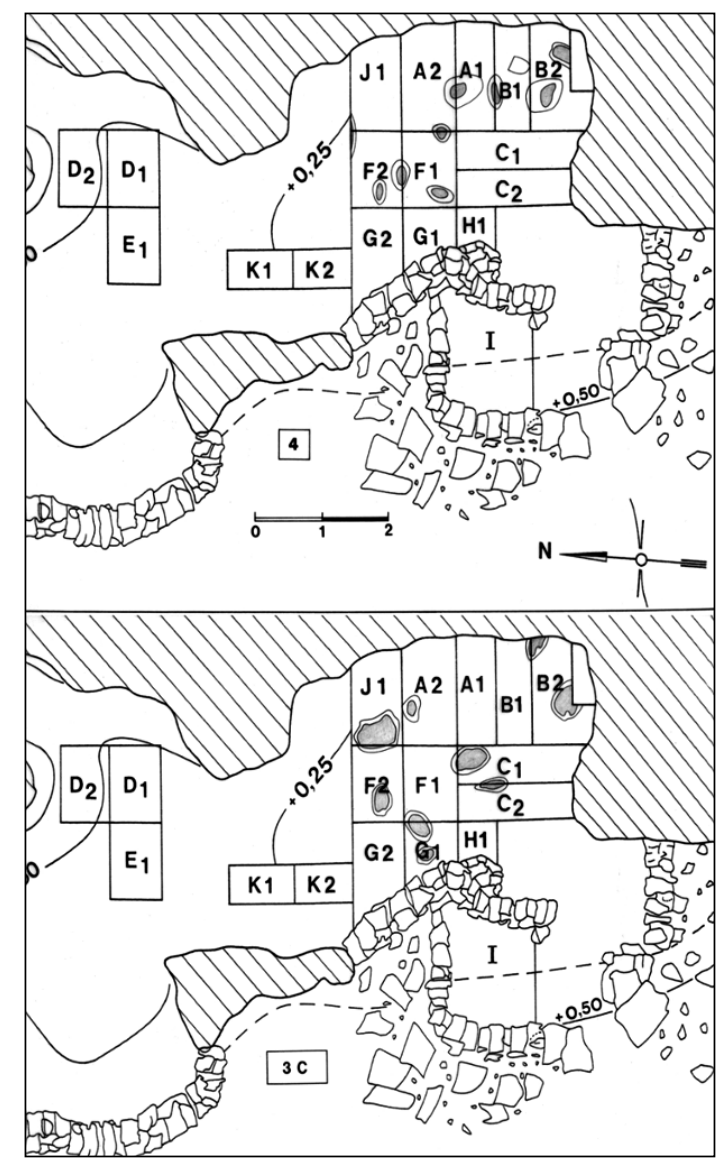

Figura 2. Distribución de los fogones en las unidades 4 (arriba) y 3C (abajo) de CDM1. Realizado por Rafael Paunero.

Distribution of hearths in units 4 (top) and 3 C (down) of CDM1. Made by Rafael Paunero.

Tabla 6. Cantidad de piezas con tratamiento y daño térmico por sector. CDM1. Unidades 4 y 3C.

Quantity of artifacts with heat treatment and thermal damage in each sector. CDM1. Units 4 and $3 C$.

\begin{tabular}{cccccccccccccccc}
\hline Sector & Alteración & A1 & A2 & B1 & B2 & C1 & C2 & E1 & F1 & F2 & G1 & J1 & K1 & L1 & Total \\
\hline Unidad 4 & Daño Térmico & 9 & 1 & 20 & 6 & 1 & 2 & 1 & 2 & - & 1 & - & 1 & - & 44 \\
& TT & 40 & 16 & 224 & 65 & 31 & 18 & 3 & 4 & 2 & - & 4 & 2 & - & 409 \\
Unidad 3C & Daño Térmico & - & 7 & 1 & 2 & 7 & 2 & - & 6 & 3 & 2 & 4 & - & - & 34 \\
& TT & - & 5 & 2 & 4 & 4 & 1 & - & 3 & 3 & 1 & 3 & - & 1 & 27 \\
\hline
\end{tabular}


Las piezas con evidencias únicamente de lustre o cambio de color (409) presentan en su mayoría lustre en ambas caras $(n=396,96,8 \%)$, mientras que las restantes tienen lustre exclusivamente en la cara ventral ( $\mathrm{n}=10,2,5 \%)$ o sólo cambiaron de color $(\mathrm{n}=3$, $0,7 \%)$. Considero que estas piezas fueron tratadas térmicamente durante la manufactura de los instrumentos líticos. En este sentido, el 79,67\% (n=286) de estos restos corresponde al estadio 3 de formatización final (sensu Skarbun et al. 2007) (Tabla 7). De estos, 73 son esquirlas de retoque $(25,5 \%)$ y 51 corresponden a adelgazamiento bifacial $(17,8 \%)$ (Tabla 8). Esto indicaría que el tratamiento térmico se habría realizado antes de la formatización final de instrumentos. Es interesante la alta cantidad de lascas de adelgazamiento bifacial alteradas, teniendo en cuenta que de los escasos instrumentos hallados en este componente, sólo dos tienen un muy leve y marginal retoque bifacial. Del total de lascas de adelgazamiento bifacial reconocidas en el conjunto (72), el 70,8\% ( $\mathrm{n}=51)$ fue tratada térmicamente. Realicé un test de Chi cuadrado para evaluar si existe dependencia entre el tipo de producto de talla del estadio 3 y la presencia o no de tratamiento térmico $\mathrm{y}$ este ha arrojado diferencias muy significativas $\left(\mathrm{X}^{2}=10,72 ; p=0,01 ;\right.$ g.l. $\left.=2\right)$. Considero que el tratamiento térmico se habría realizado sobre formas base para la confección de instrumentos bifaciales tales como puntas de proyectil, que hasta el momento no fueron recuperadas en la Unidad 4 de CDM1. Esta hipótesis se sustenta en que se ha registrado

Tabla 7. Estadio de las piezas con tratamiento térmico. Unidades 4 y $3 \mathrm{C}$.

Reduction stage of heat treated flakes. Units 4 and $3 C$.

\begin{tabular}{cccc}
\hline Unidad & 1 & 2 & 3 \\
\hline 4 & $3,06 \%$ & $17,27 \%$ & $79,67 \%$ \\
$3 \mathrm{C}$ & $5 \%$ & $20 \%$ & $75 \%$ \\
\hline
\end{tabular}

Tabla 8. Piezas del estadio 3 de formatización final con tratamiento térmico. Unidades 4 y 3C. Adelg. bifacial = Adelgazamiento bifacial. React. del filo = Reactivación del filo.

Heat treated flakes of stage 3. Units 4 and 3C. Adelg. Bifacial $=$ Bifacial thinning. React. del filo $=$ Resharpening.

\begin{tabular}{ccccccc}
\hline Unidad & $\begin{array}{c}\text { Adelg. } \\
\text { bifacial }\end{array}$ & $\begin{array}{c}\text { React. } \\
\text { del filo }\end{array}$ & Retalla Retoque & Indet. & Total \\
\hline 4 & 51 & 1 & 4 & 73 & 157 & 286 \\
$3 \mathrm{C}$ & 3 & - & - & 8 & 4 & 15 \\
\hline
\end{tabular}

en otros sitios tempranos de la región la aplicación de dicho procedimiento en puntas (Hermo 2008; Nami 1992); asimismo Nami (2010) ha realizado experimentaciones donde aplica exitosamente esta técnica en la producción de Puntas Cola de Pescado; estas son, por cronología, las puntas que sería esperable registrar en CDM1.

La longitud promedio de las lascas de adelgazamiento bifacial tratadas $(1,98 \mathrm{~cm})$ es mayor que la de este tipo de pieza sin tratamiento $(1,66 \mathrm{~cm})$. Sin embargo, al aplicar el test estadístico "t" de Student se observa que estas diferencias no son significativas $(\mathrm{t}=1,14, p=0,01 ;$ g.l. $=70)$. Así, la aplicación de este procedimiento durante el adelgazamiento bifacial no se explicaría por un aumento en la longitud de las piezas tratadas con respecto a las no tratadas, sino por otros factores. Entre las causas posibles se pueden considerar un mayor control de la talla y la menor probabilidad de cometer errores, ambos puntos importantes a la hora de producir instrumentos de difícil formatización como los artefactos bifaciales. También se podrían esgrimir cuestiones estéticas, dadas por el cambio de color y la aparición de lustre térmico, que dan a la pieza una apariencia particular. Aunque los beneficios que se dan a nivel de la facilidad de la talla son importantes, todo comportamiento tecnológico es al mismo tiempo un comportamiento político, social y simbólico (Pfaffenberger 1988). De esta manera, determinadas modificaciones en la apariencia de un artefacto pueden jugar un papel importante en la decisión de realizar o no este procedimiento, en especial en contextos con abundantes materias primas de muy buena calidad como lo es el área en estudio.

De las 409 piezas, $224(54,8 \%)$ se recuperaron de la cuadrícula B1 (Tabla 6) y 337 son de sílex rojo (82,3\%, Tabla 9, Figura 3), por lo que es probable que se trate de un evento de tratamiento térmico y talla que ha producido gran cantidad de esquirlas.

Tabla 9. Piezas con tratamiento térmico según materia prima. Unidades 4 y 3C. Calc. $=$ Calcedonia. Xil. = Xilópalo. Heat treated artifacts according to raw material. Units 4 and 3C. Calc. $=$ Chalcedony. Xil. $=$ Silicified Wood .

\begin{tabular}{cccccccc}
\hline \multirow{2}{*}{ Unidad } & \multirow{2}{*}{ Calc. } & \multirow{2}{*}{ Indet. } & \multicolumn{2}{c}{ Sílex } & \multirow{2}{*}{ Xil. } & Total \\
\cline { 4 - 6 } & & & Otros & Rojo & & \\
\hline 4 & 48 & 7 & 17 & 337 & - & 409 \\
$3 \mathrm{C}$ & 6 & - & 7 & 12 & 2 & 27 \\
\hline
\end{tabular}




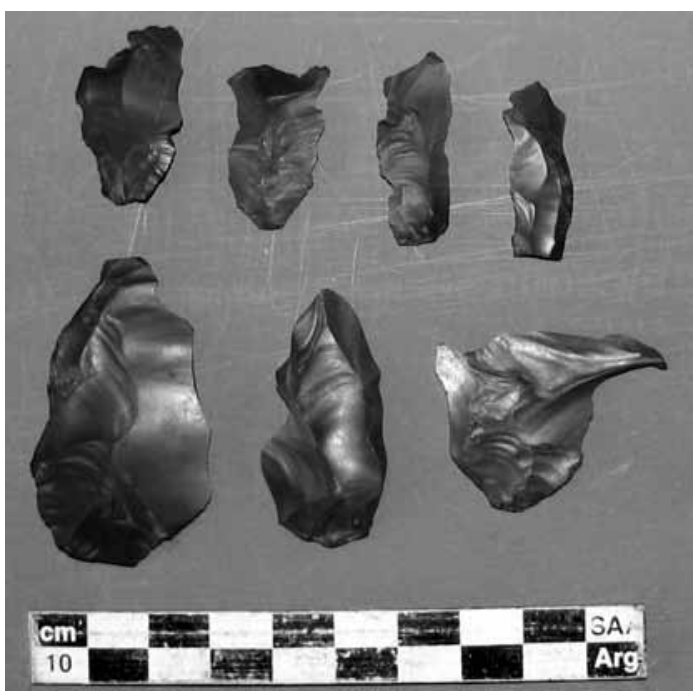

Figura 3. Lascas de adelgazamiento bifacial de sílex rojo con lustre térmico. Unidad 4.

Bifacial thinning flakes of red chert with thermal luster. Unit 4.

Si bien esto coincide con la zona de mayor concentración de restos líticos, existen otros sectores con elevado número de artefactos que prácticamente no tienen elementos tratados, como la cuadrícula F1. Además, podrían incorporarse a este conjunto tres de los seis restos dañados únicamente en la cara dorsal, que también fueron recuperados en la cuadrícula B1 y que son de sílex rojo. Entre las piezas con probable tratamiento térmico hay un cuchillo con retoque inverso extendido y bifacial distal sobre lámina y una lasca retocada de retoque inverso lateral. Ambos fueron formatizados por presión en sílex rojo.

Si bien es cierto que existe una fuerte predominancia del sílex rojo en esta unidad $(48,2 \%$, $\mathrm{n}=598$ ), ésta se acentúa al analizar los restos tratados $(82,3 \%, n=337)$. Esta materia prima es de por sí de muy buena calidad, pero mejora sus propiedades para la talla luego de este procedimiento, hecho que ha sido verificado experimentalmente (Frank et al. 2009; Nami et al. 2000). Considero factible que los antiguos ocupantes de este sitio conocieran los buenos resultados del tratamiento térmico en esta particular materia prima, y que la prefirieran por sobre otras a la hora de aplicar esta técnica. He realizado un test de Chi cuadrado evaluando si existe dependencia entre el tipo de materia prima y la presencia o no de tratamiento térmico y este ha arrojado diferencias muy significativas $\left(X^{2}=298,08 ; p=0,01 ; g . l .=4\right)$, lo que avalaría esta postura. Aunque puede llamar la atención la proporción de restos tratados de sílex rojo, debe considerarse que la mayoría de estos elementos son lascas de formatización final. Su alta concentración en sectores acotados apunta a la aplicación de este procedimiento en pocos eventos de talla, que generaron gran cantidad de desechos. Cabe destacar que, por su similitud en tonalidad con gran parte del conjunto de sílex rojo tratado, la lasca retocada tratada térmicamente podría considerarse como un subproducto generado durante uno de estos eventos de talla y aprovechado de manera expeditiva.

Por otra parte, vale la pena destacar que he desarrollado estudios en la llamada Cantera de Sílex, próxima al sitio y de la cual probablemente provenga esta materia prima. Allí no se han observado evidencias de tratamiento ni daño térmico. Se postuló que la materia prima era transportada de la cantera al sitio como formas bases, para terminar la confección de instrumental allí (Frank et al. 2007). Esto es coherente con lo propuesto más arriba referido a la aplicación del tratamiento térmico previo a la formatización final de los instrumentos líticos.

\section{Unidad 3C}

La Unidad 3C presenta abundantes productos de talla $(n=1.190)$, pocos instrumentos $(n=7)$ y ningún núcleo. A diferencia de la Unidad 4, predominan los productos de talla del estadio de talla (estadio 2 , $\mathrm{n}=381$ ) por sobre los de formatización final (estadio $3, \mathrm{n}=233$ ). Son escasos los de descortezamiento (estadio 1, $\mathrm{n}=27$, Tabla 2). La materia prima predominante es el sílex (92\%, $\mathrm{n}=1.103)$, en especial el de color rojo de procedencia local $(77,5 \%$ del total del conjunto, $n=928$ ) (Tabla 3 ).

En esta unidad se reconocieron 61 piezas termoalteradas. Esto corresponde al 5\% del total del conjunto lítico de la unidad. De ellas, 34 muestran daño térmico y las 27 restantes cambios únicamente en color, lustre o pátina.

Todas las piezas dañadas presentan longitudes menores a $3,4 \mathrm{~cm}$, y la mayoría son esquirlas $(44,1 \%$, $\mathrm{n}=15$, Tabla 4). Además, de estas 34 piezas, 23 (68\%) presentan daños en ambas caras, por lo que su termoalteración se produjo después de haber sido extraídas del soporte original. Lo mismo sucede con los seis restos dañados exclusivamente en su cara ventral (Tabla 10). Estas características me llevan a descartar, para la mayor parte de estas piezas, el daño producto del tratamiento térmico. Únicamente 
Tabla 10. Piezas con rasgos de alteración térmica. Unidad 3C. $\mathrm{No}=\mathrm{Sin}$ daño térmico pero con alteraciones del tipo lustre, color y pátina.

Artifacts with indications of thermal alteration. Unit $3 C$. No = Without heat damage but with other alterations such as luster, color and patina.

\begin{tabular}{cccc}
\hline \multirow{2}{*}{ Daño Ventral } & \multicolumn{2}{c}{ Daño Dorsal } & \multirow{2}{*}{ Total } \\
\cline { 2 - 3 } & No & Sí & \\
\hline No & 27 & 5 & 32 \\
Sí & 6 & 23 & 29 \\
Total & 33 & 28 & 61 \\
\hline
\end{tabular}

aquellas esquirlas con daño térmico sólo en su cara dorsal (cinco) pueden ser producto del tratamiento térmico de una pieza y su posterior talla.

$\mathrm{Al}$ analizar su procedencia, se observa que las piezas dañadas provienen mayormente de cuadrículas en las que se han registrado fogones (Tabla 6, Figura 2). La termoalteración de estas piezas se debe probablemente a la caída accidental de lascas en los fogones, al momento de confección de instrumental lítico, o posteriormente. Al igual que en la Unidad 4, dejo de lado otras posibilidades como un descarte intencional de las piezas a los fogones por la baja cantidad de lascas dañadas en relación al total del sitio. Además se presentan dañados únicamente productos de talla, por lo que no responde a las expectativas ni de actividades de mantenimiento ni en el marco de rituales mortuorios. También descarto la posibilidad de alteración térmica por fuegos encendidos en ocupaciones posteriores, ya que en la Unidad 3B no se han reconocido fogones. Sin embargo, las lascas se presentan relativamente dispersas, por lo que es probable que el conjunto no refleje la distribución original al momento de la termoalteración. Esto deberá ser comprobado con futuros estudios.

Las piezas con evidencias únicamente de lustre o color (27) presentan en su mayoría lustre en ambas caras $(93 \%, \mathrm{n}=25)$, mientras que las restantes tienen lustre en la cara ventral $(7 \%, \mathrm{n}=2)$. Sólo en dos lascas he identificado cambio de color. Aunque es probable que estas piezas hayan sido tratadas térmicamente durante la manufactura de instrumental lítico, las características del subconjunto no arrojan resultados precisos al respecto. La cantidad de piezas con lustre y cambio de color es muy escasa, aún si se consideran las lascas con daño térmico en la cara dorsal. Aunque el $75 \%$ de los restos $(n=15)$ corresponden al estadio 3 de formatización final (Tablas 7 y 8), a diferencia de lo que ocurre en la Unidad 4, en esta unidad se han registrado evidencias de tratamiento térmico en gran cantidad de cuadrículas (10) y distribuidas relativamente de manera uniforme (Tabla 6). Además, he registrado evidencias de tratamiento térmico en cinco diferentes materias primas, de modo que cada una presenta muy escasos restos termoalterados (Tabla 9). Aún así, el sílex rojo es el que presenta mayor cantidad de restos tratados $(44,4 \%, n=12)$. Es posible entonces que esté registrando diferentes casos de tratamiento térmico, de los cuales se han recuperado escasas piezas. En este sentido es necesario resaltar que para este componente se han recuperado más productos de talla del estadio 2 (de talla) que del 3 (de formatización final). Así, los resultados obtenidos para este componente apuntan, al igual que la información derivada de la Unidad 4, a que este procedimiento se realizaría antes de la formatización final de los instrumentos.

\section{Conclusiones}

Los componentes tempranos de CDM1 presentan evidencias de alteración térmica de artefactos líticos en diverso grado. Esta alteración se debió al tratamiento térmico de piezas durante el proceso de producción de artefactos líticos y, en menor medida, a otras causas tales como el contacto accidental de los artefactos con los fogones.

Con respecto a los restos tratados térmicamente, su presencia es muy importante en la Unidad 4. En este componente destaca la gran cantidad de lascas de adelgazamiento bifacial con lustre térmico; además se observa una predominancia de piezas de sílex rojo entre los elementos tratados.

Este procedimiento se habría realizado antes de la formatización final, en el proceso de confección de instrumental bifacial, como por ejemplo puntas de proyectil, a pesar de que éstas no han sido recuperadas en el sitio. Este instrumental habría sido confeccionado sobre sílex rojo, materia prima de muy buena calidad, pero que mejora sus propiedades para la talla luego del tratamiento térmico. En este sentido, considero que existía a fines del Pleistoceno un conocimiento acerca de la utilidad de este procedimiento en esta materia prima, y en consecuencia un uso preferencial de ésta en los casos en que se practicara la técnica de tratamiento térmico. Las lascas de adelgazamiento bifacial tratadas no son más largas que las no tratadas. Esto 
me lleva a pensar que las causas de aplicación de este procedimiento estarían en otros factores. Creo, en este sentido, que podrían influir otras variables relacionadas con un aumento en la aptitud para la talla (mayor control de las extracciones, menor fuerza necesaria, menor probabilidad de falla) así como cuestiones estéticas (dadas por el cambio en lustre y color de las piezas).

En ninguno de los componentes estudiados se han reconocido núcleos, por lo que resulta imposible saber si estas piezas fueron tratadas. Los análisis de una cantera taller de sílex rojo no han arrojado evidencias de tratamiento térmico, y además brindan información acerca del transporte de formas base hacia los sitios de actividades múltiples. Esta evidencia, junto con mis observaciones experimentales, me lleva a postular el tratamiento térmico de formas base para los sitios de la región.

Por otra parte, al observar la distribución de los restos tratados térmicamente noto que éstos se concentran principalmente en las cuadrículas B, en especial la B1. Considero que estoy registrando un evento de talla bifacial sobre sílex rojo tratado térmicamente, realizado en los alrededores del fogón registrado en la cuadrícula B1. Por supuesto, es posible que ciertos factores postdepositacionales afectaran la distribución del conjunto, concentrando estos elementos en esa zona. Si bien esto debe ser analizado con mayor profundidad, lo considero poco probable, teniendo en cuenta la horizontalidad de la unidad así como la ausencia de evidencia de alteración por roedores, raíces u otros elementos disturbantes.

Los resultados obtenidos en la Unidad $3 \mathrm{C}$ no son tan claros como los de la Unidad 4. Aunque algunos artefactos presentan las características típicas del tratamiento térmico, se observan muy escasos artefactos con estos rasgos en relación al total del conjunto lítico. Además, estas piezas se presentan en baja cantidad en un número elevado de materias primas. También se debe considerar su alta dispersión, lo que queda evidenciado por la gran cantidad de cuadrículas en que se recuperaron estos elementos. En este sentido, es posible que se haya realizado este procedimiento pirotecnológico en la cueva, pero este componente presenta características diferentes a la Unidad 4, que vuelven difícil afirmarlo fehacientemente. Si se tiene en cuenta que en este componente las actividades están más centradas en la confección de formas base que en la formatización final de los instrumentos, la información es coherente con lo propuesto anteriormente acerca de que el tratamiento térmico se realizaría hacia los momentos finales de la producción lítica.

Considero que la baja cantidad de elementos posiblemente tratados, sumado a la alta variedad de materias primas y su gran dispersión, podrían indicar que el tratamiento térmico y posterior talla de estos artefactos se habría realizado en una zona ajena al área excavada, dentro del sitio o quizás en otro sitio, y que los restos recuperados tratados habrían llegado allí posteriormente por diversas causas. Esto coincide con lo propuesto por Paunero et al. (2007) quienes sugieren que en esta unidad el espacio se articuló de manera distinta a lo evidenciado en el componente 4 , teniendo en cuenta la distribución de fogones, artefactos líticos y elementos óseos. Según estos autores, “... los indicadores señalan probables áreas de actividades diferentes y complementarias, por un lado, la talla lítica y por otro, el procesamiento de fauna..." (Paunero et al. 2007:583).

Por las características tipológicas y la ubicación de los indicadores, el daño térmico no se produjo durante el tratamiento térmico. Las piezas dañadas representan siempre un porcentaje muy bajo del conjunto, lo que permite desechar su descarte al fuego, al menos como práctica habitual. Por otra parte, la mayoría de las piezas dañadas provienen de cuadrículas con fogones. En consecuencia, considero que es factible que el daño térmico de estos artefactos se haya producido por su caída accidental en estas estructuras de combustión.

Agradecimientos: Agradezco a Rafael Paunero, Alicia Castro y Fabiana Skarbun por sus consejos. A mis compañeros de equipo por su apoyo. A tres evaluadores anónimos, cuyos comentarios enriquecieron el trabajo. Una versión preliminar de este trabajo fue realizada para el Seminario de Doctorado "Tecnología Lítica: Discutiendo sobre Materias Primas" dictado en la Facultad de Filosofía y Letras (UBA) por la Dra. P. Escola a quien agradezco por sus comentarios. A la familia Behm por su cariño y ayuda en el campo. Este trabajo fue parcialmente financiado por una beca doctoral del CONICET. 


\section{Referencias Citadas}

Aschero, C. 1975. Ensayo para una clasificación morfológica de artefactos líticos aplicada a estudios tipológicos comparativos. Informe presentado al CONICET, Buenos Aires. Buenos Aires.

Bordes, F. 1969. Traitement thermique du silex au Solutréen. Bulletin de la Société Préhistorique Française 66:197.

Cardich, A. y N. Flegenheimer 1978. Descripción y tipología de las industrias más antiguas de Los Toldos. Relaciones de la Sociedad Argentina de Antropología XII:225-242.

Cardich, A., M. Mansur, M. Giesso y V. Duran 1981-1982. Arqueología de las cuevas de El Ceibo (Pcia. de Santa Cruz, Argentina). Relaciones de la Sociedad Argentina de Antropología XIV:241-267.

Cattáneo, R., A. Pupio, M. Valente y A. Barna 1997-1998. Alteración térmica en dos tipos de rocas silíceas: resultados experimentales y aporte de datos para el análisis arqueológico. Relaciones de la Sociedad Argentina de Antropología XXII-XXIII:343-361.

Clemente Conte, I. 1995. Sílex y lustre térmico en el Paleolítico Medio ¿alteración o técnica de talla? El ejemplo de Mediona 1. (Alt. Penedes Barcelona). Actas de Trabalhos de Antropologia y Etnologia 35:37-43.

Collins, M. y J. Fenwick 1974. Heat treating of chert: Methods of interpretation and their application. Plains Anthropologist 19:134-145.

Cueto, M. y A. Frank 2008. Tratamiento térmico de artefactos líticos: estudios experimentales. En Problemáticas de la Arqueología Contemporánea, compilado por A. Austral y M. Tamagnini, Tomo II, pp. 375-384. Universidad Nacional de Río Cuarto, Río Cuarto.

Frank, A.D. 2009. El daño térmico en artefactos líticos: estudios experimentales. En Entre Pasados y Presentes II. Estudios Contemporáneos en Ciencias Antropológicas, editado por T. Bourlot, D. Bozzuto, C. Crespo, A.C. Hecht y N. Kuperszmit, pp. 35-48. Fundación de Historia Natural Félix de Azara, Buenos Aires.

Frank, A.D., M. Cueto, A. Castro y R. Paunero 2009. El impacto del tratamiento térmico en las huellas de uso en sílices de la Meseta Central de Santa Cruz. Estudios experimentales. Manuscrito en posesión del autor.

Frank, A.D., F. Skarbun y M.F. Paunero 2007. Hacia una aproximación de las primeras etapas de reducción lítica en el Cañadón de la Mina, Localidad Arqueológica La María, Meseta Central de Santa Cruz Argentina. Magallania 35:133-144.

Gassin, B., V. Léa, J. Linton y L. Astruc 2006. Production, gestion et utilisation des outillages lithiques du Chasséen méridional. Normes Techniques et Pratiques Sociales de la Simplicité des Outillages Pré- et Protohistoriques. Actes de XXVIe Recontres Internationals d'Archaeologie et d'Historie d'Antibes, pp. 223233. Éditions APDCA, Antibes.

Hermo, D. 2008. Rocas como símbolos: la selección de materias primas para puntas de proyectil en ambientes mesetarios de Patagonia. Intersecciones en Antropología 9:319-324.
Inizan, M.L., M. Reduron-Ballinger, H. Roche y J. Tixier 1999. Raw materials. En Technology and Terminology of Knapped Stone. Préhistoire de la Pierre Taillée, editado por M.L. Inizan, M. Reduron-Ballinger, H. Roche y J. Tixier, Tomo 5, pp. 19-28. CREP, Nanterre.

Inizan, M.L. y J. Tixier 2000. L'émergence des arts du feu: le traitement thermique des roches siliceuses. Paléorient 26:23-36.

Larsson, L. 2000. The passage of axes: fire transformation of flint objects in the Neolithic of southern Sweden. Antiquity 74:602-610.

Luedtke, B. 1992. An Archaeologist's guide to chert and flint. Archaeological Research Tools 7. Institute of Archaeology, UCLA.

Mandeville, M. 1973. A consideration of the thermal pretreatment of chert. Plains Anthropologist 18:177-202.

Musters, G. 2003. Vida entre los Patagones. El Elefante Blanco, Buenos Aires.

Nakazawa, Y. 2007. Hearth-Centered Spatial Organization: A Comparative Approach to the Study of Palimpsests in late Upper Paleolithic Sites in Hokkaido (Japan) and Cantabria (Spain). Tesis Doctoral, University of New Mexico, Albuquerque.

Nami, H. 1992. El subsistema tecnológico de la confección de instrumentos líticos y la explotación de los recursos del ambiente: una nueva vía de aproximación. Shincal 2:13-53.

- - - 2010. Tecnología paleoindia de Sudamérica: nuevos experimentos y observaciones para investigar la secuencia de reducción Fell. Orígenes 9:1-40.

Nami, H., G. Cattáneo y M. Pupio 2000. Investigaciones experimentales sobre el tratamiento térmico en algunas materias primas de Pampa y Patagonia. Anales del Instituto de la Patagonia 28:315-329.

Orquera, L. y E. Piana 1986. Normas para la descripción de objetos arqueológicos de piedra tallada. CADIC Contribución Científica. Publicación Especial 1, Ushuaia.

Patterson, L. 1984. Comments on studies of thermal alteration of Central Pennsylvania jasper. American Antiquity 49:168-173.

Paunero, R.S. 2009. Fogones, conjuntos líticos y funcionalidad en el componente pleistocénico del sitio Cueva 1 de Cerro Tres Tetas, provincia de Santa Cruz. En Arqueología Argentina en los inicios de un nuevo siglo, editado por F. Oliva, N. de Grandis y J. Rodríguez, Tomo 2, pp. 419-430. Universidad Nacional de Rosario, Rosario.

Paunero, R.S. y A. Castro 2001. Análisis lítico y funcionalidad del componente inferior del sitio Cueva 1, localidad arqueológica Cerro Tres Tetas, provincia de Santa Cruz, Argentina. Anales del Instituto de la Patagonia (Serie Ciencias Sociales) 29:189-206.

Paunero, R.S., A.D. Frank, F. Skarbun, G. Rosales, M. Cueto, G. Zapata, M. Paunero, N. Lunazzi y M.D. Giorgio 2007. Investigaciones arqueológicas en sitio Casa del Minero 1, Estancia La María, meseta central de Santa Cruz. En Arqueología de Fuego-Patagonia. Levantando Piedras, Desenterrando Huesos... y Develando Arcanos, editado por F. Morello, A. Prieto, M. Martinic y C. Bahamonde, pp. 577-588. CEIDER, Punta Arenas. 
Pfaffenberger, B. 1988. Fetishised objects and humanised nature: Towards an anthropology of technology. Man (New Series) 23:236-252.

Purdy, B. y H. Brooks 1971. Thermal alteration of silica minerals: An archaeological approach. Science 173:322-325.

Rick, J. y S. Chappell 1983. Thermal alteration of silica materials in technological and functional perspective. Lithic Technology 12:69-80.

Schindler, D., J. Hatch, C. Hay y R. Bradt 1982. Aboriginal thermal alteration of a Central Pennsylvania jasper: Analytical and behavioral implications. American Antiquity 47:526-544.

Skarbun, F. 2009. La Organización Tecnológica en Grupos Cazadores Recolectores desde las Ocupaciones del Pleistoceno
Final al Holoceno Tardio, en la Meseta Central de Santa Cruz. Tesis doctoral, FCNyM, Universidad de la Plata, La Plata.

Skarbun, F., A. Frank, M. Paunero, M. Cueto y G. Rosales 2007. Análisis de la tecnología lítica del sitio Casa del Minero 1, meseta central de Santa Cruz. En Arqueología de Fuego-Patagonia. Levantando Piedras, Desenterrando Huesos... y Develando Arcanos, editado por F. Morello, A. Prieto, M. Martinic y C. Bahamonde, pp. 589-600. CEIDER, Punta Arenas.

Stadler, N. 2002. El Uso del Tratamiento Térmico sobre las Materias Primas Líticas en el Área del Lago Argentino. Tesis de Licenciatura, FFyL, Universidad de Buenos Aires, Buenos Aires.

Terradas, X. y J.F. Gibaja 2001. El tratamiento térmico en la producción lítica: el ejemplo del Neolítico catalán. Cypsela 13:31-58. Museu d'Arqueologia de Catalunya.

\section{Nota}

1 Los productos de talla son todos aquellos artefactos líticos originados antrópicamente y generados como consecuencia del proceso de reducción de núcleos y de la manufactura y mantenimiento de los instrumentos, mediante presión o percusión (Paunero y Castro 2001; Skarbun 2009; Skarbun et al. 2007). No empleo el concepto "desechos de talla" puesto que en él subyace la idea de que estos elementos no son utilizados. Por el contrario, estos son potencialmente útiles y su uso sólo puede ser contrastado a través de otras vías de investigación, como el análisis funcional. 
